\title{
Microwave emulations and tight-binding calculations of transport in polyacetylene
}

\author{
Thomas Stegmann $^{\mathrm{a}}$, John A. Franco-Villafañe ${ }^{\mathrm{b}, \mathrm{a}}$, Yenni P. Ortiz ${ }^{\mathrm{a}}$, Ulrich Kuhl ${ }^{\mathrm{c}}$, Fabrice Mortessagne ${ }^{\mathrm{c}}$, Thomas H. Seligman ${ }^{\mathrm{a}, \mathrm{d}}$ \\ ${ }^{a}$ Instituto de Ciencias Físicas, Universidad Nacional Autónoma de México, Avenida Universidad s/n, 62210 Cuernavaca, México \\ ${ }^{b}$ Instituto de Física, Benemérita Universidad Autónoma de Puebla, Apartado Postal J-48, 72570 Puebla, México \\ ${ }^{c}$ Université de Nice - Sophia Antipolis, Laboratoire de la Physique de la Matière Condensée, CNRS, Parc Valrose, O6108 Nice, France \\ ${ }^{d}$ Centro Internacional de Ciencias, 62210 Cuernavaca, México
}

\section{Abstract}

A novel approach to investigate the electron transport of cis- and trans-polyacetylene chains in the single-electron approximation is presented by using microwave emulation measurements and tight-binding calculations. In the emulation we take into account the different electronic couplings due to the double bonds leading to coupled dimer chains. The relative coupling constants are adjusted by DFT calculations. For sufficiently long chains a transport band gap is observed if the double bonds are present, whereas for identical couplings no band gap opens. The band gap can be observed also in relatively short chains, if additional edge atoms are absent, which cause strong resonance peaks within the band gap. The experimental results are in agreement with our tight-binding calculations using the nonequilibrium Green's function method. The tight-binding calculations show that it is crucial to include third nearest neighbor couplings to obtain the gap in the cis-polyacetylene.

Keywords: coherent transport, polyacetylene, microwave emulation experiments, nonequilibrium Green's function method PACS: 73.63.-b, 73.23.-b

\section{Introduction}

The aim of further miniaturization of electronic devices has led in recent years to the question, if it is possible to shrink down the individual active element to a single molecule. This question stimulated the research field of molecular electronics, see [1] and references therein for an overview. One approach is based on carbon nanotubes, which for certain geometric parameters (i.e. tube diameter or number of windings) show a band gap and hence, can be used as transistors [2, 3, 4, 5]. A recent milestone has been the realization of a carbon nanotube computer [6]. However, a drawback of this approach is that after the growth of the nanotubes the metallic carbon nanotubes have to be separated from the semi-conducting ones. An alternative approach could be to use individual polyacetylene chains, see a sketch in figure $1(a, b)$, which are predicted to have a band gap [7, 8, 9, 10, 11, 12]. However, most of the experimental work has been done with thin films of polyacetylene chains [13, 14]. Transport experiments with individual polyacetylene chains have, to the best of our knowledge, not been performed yet. However, new developments in microwave experiments allow to emulate experimentally a tight-binding model, which has proven successful in studies of graphene [15, 16]. These microwave experiments, which are performed here for the first time on molecular structures, can measure the transport of microwaves through one or two dimensional tight-binding systems. The microwave transmission corresponds to the ballistic single electron transport in mesoscopic physics or in molecules. Electron-electron interaction, which is present to some extent in real molecules, cannot be emulated by the microwave experiment. (a)

(b)

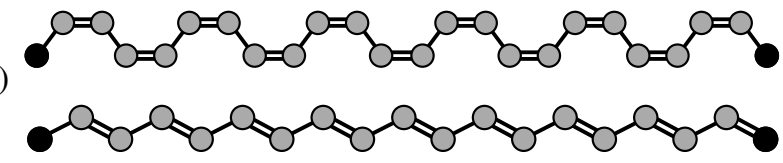

(c)

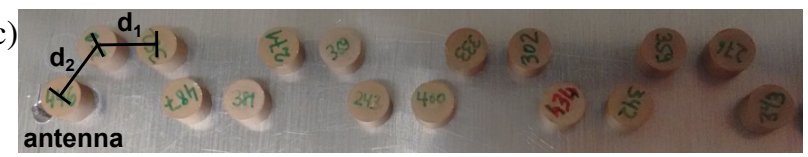

(d)

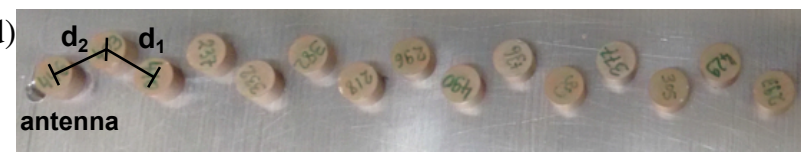

Figure 1: Structure of polyacetylene chains in cis-geometry (a) with $N_{d}=11$ dimers and $N_{c}=2$ additional edge atoms, which are not part of the dimers, and in trans-geometry (b) with $N_{d}=9$ dimers and a single edge atom $\left(N_{c}=1\right)$ at the left chain end. The resonators correspond to carbon atoms, the hydrogen atoms are not shown. The blue shaded resonators at the chain ends indicate the resonators to which the contacts (or antennas) are coupled to study the transport. The cis-chain corresponds to the armchair shape, whereas the trans-chain corresponds to a zigzag shape. In (c) and (d) photos of the microwave experiment to emulate polyacetylene chains for the cis- and trans-geometry are shown, respectively. On the left hand side the antenna on the bottom plate is seen, whereas the antenna on the right hand side is mounted to the top plate (not shown).

In this paper we explore the transport in polyacetylene like systems. In long chains a transport gap is observed, which is expected due to the dimerization of the chain, i.e. the chain is composed of unit cells of two carbon atoms (dimers). Additional edge atoms add edge localized resonance states within the gaps, which become important for short chains but are neg- 

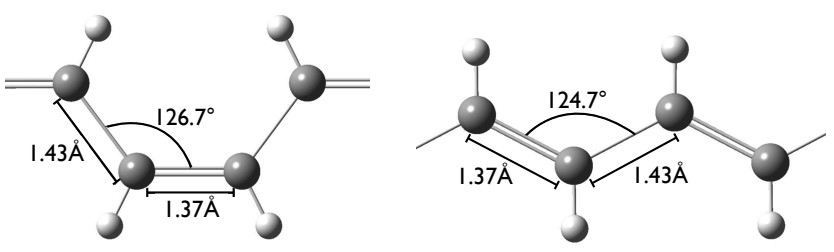

Figure 2: Stable structure of the polyacetylene molecule, optimized by the DFT method. The left part shows the cis-configuration (armchair) and the right part the trans-configuration (zigzag) with the corresponding angles and distances between the carbon atoms. The larger gray spheres correspond to the carbon atoms and the smaller white spheres to the hydrogen atoms, which saturate the dangling bonds of the carbon atoms.

ligible for long chains.

\section{System: Polyacetylene chains}

The setup, which we use to emulate both the cis- (armchair) and trans- (zigzag) isomers of polyacetylenes of various lengths, is shown in figure 1 (c,d). The studied chains consist of $N_{d}$ dimers, which we here define via the double bonds, with $N_{c}$ additional edge atoms at the chain ends, which do not belong to dimers. The chains have altogether $N=2 N_{d}+N_{c}$ atoms. For example, a cis-chain consisting of $N_{d}=11$ dimers with $N_{c}=2$ additional edge atoms, one at each end of the chain, is shown in fig1(a). A trans-chain of $N_{d}=9$ dimers with a single edge atom on the left can be seen in figure 1 (b).

\subsection{DFT studies}

At first, we have calculated the optimal structure of the molecule by means of density functional theory (DFT). The key idea of DFT is that the ground-state of an interacting manybody system is a unique functional of the electron density [17, 18]. The ground-state is found by minimizing its energy with respect to the electron density. In order to perform the minimization of the ground-state energy functional, an atomic basis and an exchange-correlation potential have to be selected. The atomic basis is used to construct attempts to the ground-state, while the exchange-correlation potential is taking into account the Coulomb interaction between the electrons. It is known exactly only for the free electron gas, but accurate approximations have been developed. This reduces the interacting many-body problem to a system of non-interacting particles in an effective potential. Here, we use the 6-311g(d) basis set [19], which consists of Gaussian functions, and the B3LYPD hybrid exchangecorrelation functional [20]. The calculations are performed by the DFT program GAUSSIAN09 [21]. A detailed introduction into DFT can be found for example in [22, 23].

Polyacetylenes are chains of carbon atoms. The dangling bonds of the carbon atoms are saturated by hydrogen atoms which are taken into account in our DFT studies, see figure 2. However, as their contribution to the conductance can be neglected, the hydrogen atoms will not be considered in the microwave experiment nor in the tight-binding model, see figure 1 In both cases, the most stable structure is found for dimerized chains, where the nearest-neighbor distance of the carbon atoms alternates between two different values. For the cis-chain, the horizontal bonds have a length of $1.37 \AA$ while the diagonal bonds have a length of $1.43 \AA$ with an angle of $126.7^{\circ}$, see figure 2 (left). For the trans-chain, the bond lengths are $1.37 \AA$ and $1.43 \AA$ with an angle of $124.7^{\circ}$, see figure 2 (right). Our findings agree with other $\mathrm{ab}$ initio studies of these molecules [24, 25]. This dimerization, which is also known as Peierls distortion [26], is indicated in figure 1 and figure 2 by alternating single and double bonds between the carbon atoms. Homogenous chains, where the nearest-neighbor distance of all carbon atoms is the same, do not give the energetically optimal structure.

\subsection{Microwave experiment}

Using the techniques, developed to investigate the band structure of graphene [15, 16] and to emulate relativistic systems [27, 28], we have performed an analogous experiment with microwave resonators to study the transport properties of the chains. A set of identical dielectric cylindrical resonators ( $5 \mathrm{~mm}$ height, $4 \mathrm{~mm}$ radius, refractive index $n \approx 6$ ) is placed between two metallic plates. The nearest neighbor distance of the resonators is $d_{1}=12.0 \mathrm{~mm}$ for the long bonds and $d_{2}=11.5 \mathrm{~mm}$ for the short bonds, giving the same distance ratio $d_{1} / d_{2}$ as in the DFT calculations. Close to the chain ends, see the blue shaded resonators in figure 1, antennas are placed through which microwaves (transverse electrical (TE-)modes) can be injected and detected. The individual resonators have an isolated resonance at $v_{0}^{\prime}=6.65 \pm 0.005 \mathrm{GHz}$. We restrict our investigation to frequencies around $v_{0}^{\prime}$, where each resonator contributes only one resonance. From now on we will use the normalized frequency $v=v^{\prime}-v_{0}^{\prime}$, where the resonance frequency of the resonators is at $0 \mathrm{MHz}$. Photos of the experimental setup (without the metallic plate on top) are shown in figure 1 (c,d), and a detailed description can be found in [16].

\subsection{Tight-binding model}

Theoretically, we model the polyacetylene chains by the tight-binding Hamiltonian

$$
H=\sum_{|i-j| \leq d} t_{|i-j|}|i\rangle\langle j|,
$$

which provides a qualitative good description of the electronic transport in polyacetylene chains [7, 8, [12, 29, 30]. A more complete understanding can certainly be gained by taking into account electron correlations [31, 32], yet the tight-binding model is often a good starting point for studies of molecular electronics [1].

The location of the isolated spectral peaks for the smallest cis- and trans-chains in our experiments are used to fit the onsite energies and the coupling parameters, i.e. the part of the spectrum for the smallest molecules which is uniquely determined by our measurement is used to fix the non-zero couplings for each of the two chains. This is in keeping with the standard technique of extracting a Hamiltonian from a "polyad" for molecules [33, 34]. Note, that we actually have polyads 


\begin{tabular}{c|c|c|c|c}
\hline cis-chain (armchair) & $t_{1}$ & $t_{1}^{\prime}$ & $t_{2}$ & $t_{3}$ \\
\hline & 43.9 & 36.2 & 3.2 & 3.1 \\
\hline$t_{t_{2}}^{t_{1}^{\prime}}=0$ & 43.9 & 36.2 & 4.2 & -
\end{tabular}

Table 1: Coupling strength (in MHz), which are used to fit our calculation to the experiments. The couplings between first nearest neighbors are identical for both types of chains. The two isomers differ in the coupling strengths between second and third nearest neighbors. Interactions to higher nearest neighbors, see dashed lines in the figures, can be ignored due to their large distance and the screening of surrounding atoms. Notice that $t_{3}$ is not existing in the transchain (zigzag) configuration.

here because similar other spectra will appear for different frequency ranges, yet they are well separated from the spectra we study. The thus obtained coupling strengths $t_{i}$ are listed in table 1 and depicted in figure 3 The sum in (1) takes into account interactions between the resonators up to the 3 rd nearest neighbors (definition see table 11). Have in mind that we define the $n$ 'th nearest neighbor by its index difference $n=|i-j|$ along the chain and not by the real distances $d_{i j}=\left|\vec{r}_{i}-\vec{r}_{j}\right|$. As the distances $d_{1}$ and $d_{2}$ of first nearest neighbors (1nn) are identical in both types of chains, see figure 2, also their couplings $t_{1}$ and $t_{1}^{\prime}$ are the same for both isomers. As the nearest neighbor interactions are the dominant ones the measured and calculated transmission shows qualitatively the same main features for cis(armchair) or trans-(zigzag) geometry. However, subtle differences can be noticed due to interactions between resonators, which are farther away. Due to the different bond angles in the two isomers, see figure 2 the couplings between second nearest neighbors $\left(2 \mathrm{nn}, t_{2}\right)$ are different. Polyacetylene chains have two types of third nearest neighbors. Third nearest neighbors of the first type can be neglected due to their large distance and the strong screening by the closer resonators, see the dashed lines in the figures of table 1 . Third nearest neighbors of the second type ( $\left.t_{3}\right)$ exist only in cis-chains (armchair) and cannot be neglected to understand the experimental data. Higher nearest neighbors couplings have been ignored in both chains as well. In section 3.2. we discuss effects on the transmission which would be observed if, for example, the third nearest neighbors in the cis-chains would not be taken into account. The coupling strength decays approximately exponentially with the distance of the resonators [16], see figure 3 . In first approximation, an exponential decay is also expected in real polyacetylene chains, where the coupling strength is determined by the overlap of the atomic wave functions.

\subsection{NEGF method}

In order to calculate the transmission through the chains, we apply the non-equilibrium Green's function (NEGF) method [35, 1]. The Green's function of the chain is defined as

$$
G=\left[v-H-\Sigma_{1}-\Sigma_{N}-\Sigma_{\mathrm{abs}}-\Sigma_{\mathrm{dis}}\right]^{-1}
$$

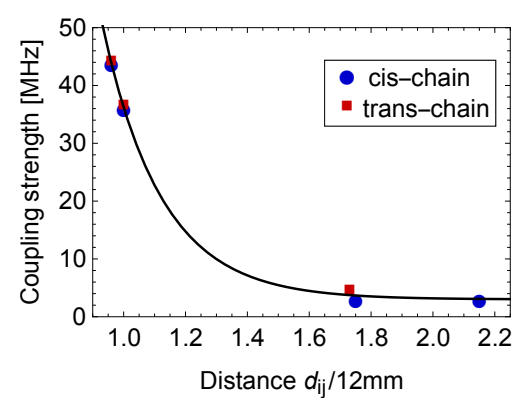

Figure 3: The coupling strength between between the resonators decays approximately exponentially with the distance $d_{i j}$ of the resonators. The solid line is given by $t=6031 \mathrm{MHz} \exp \left(-5.2 d_{i j} / 12 \mathrm{~mm}\right)+2.99 \mathrm{MHz}$.

where $v$ is the microwave frequency or, in analogy, the electron energy. The influence of the antennas (or source and drain contacts) coupled to the chain ends (blue shaded atoms number 1 and $N$ in figure 11 is taken into account by an imaginary self-energy

$$
\Sigma_{1}=-\mathrm{i} \eta|1\rangle\langle 1| \quad \text { and } \quad \Sigma_{N}=-\mathrm{i} \eta|N\rangle\langle N|,
$$

where the coupling strength $\eta=1 \mathrm{MHz}$ is adjusted to the experiment. The absorption, which is present in the experiment, is modeled by the imaginary potential (or self-energy)

$$
\Sigma_{\mathrm{abs}}=-\mathrm{i} \gamma(N) \sum_{i=1}^{N}|i\rangle\langle i| .
$$

We obtain best agreement between the experiments and the calculations assuming a linear decay of the absorption

$$
\frac{\gamma(N)}{\mathrm{MHz}}=1.17-0.013 N
$$

In the experiment, some degree of disorder cannot be avoided completely due to the uncertainty of the resonance frequency of the resonators and the uncertainty of their positions. In the calculations, disorder is taken into account by a random potential (or self-energy)

$$
\Sigma_{\text {dis }}=\sum_{i=1}^{N} \epsilon_{i}|i\rangle\langle i|,
$$

where the $\epsilon_{i}$ are chosen from a Gaussian distribution which is cut at its full width half maximum, which corresponds approximately to the experimentally observed distribution and the used selection rule. We consider the standard deviation $\sigma=5 \mathrm{MHz}$ and an ensemble of $10^{3}$ realizations.

The transmission between source and drain is then given by

$$
T(v)=4 \operatorname{Tr}\left(\operatorname{Im}\left(\Sigma_{1}\right) G \operatorname{Im}\left(\Sigma_{N}\right) G^{\dagger}\right)
$$

Note that in absence of interactions, as in this study, the NEGF method is equivalent to a scattering or transfer matrix method [35]. 

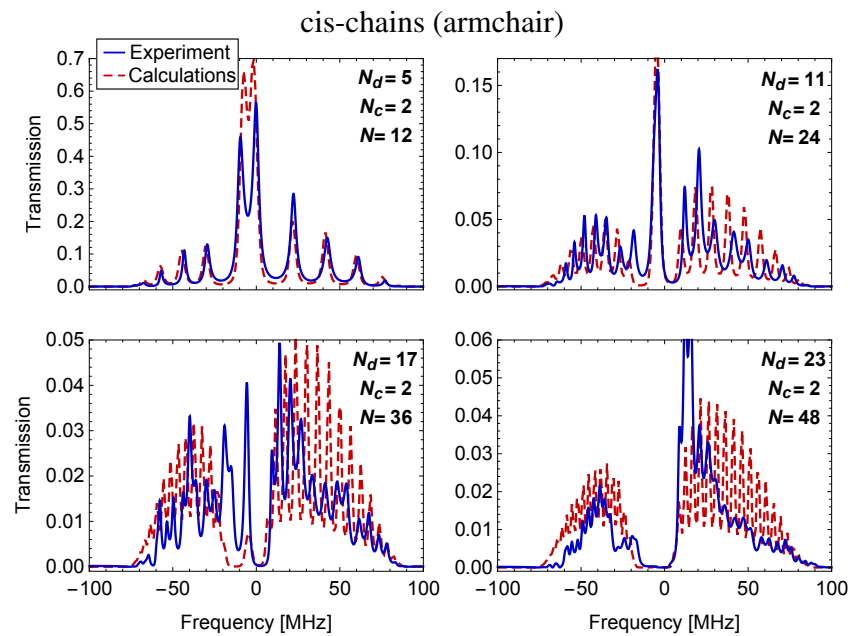

Figure 4: Transmission as a function of the microwave frequency through cispolyacetylene chains (armchair) of increasing length $N$. The blue-solid curves give the experimental data while the red-dashed curves represent the Green's function calculations. The short chains show distinct peaks, in particular around the frequency $v=0$. With increasing chain length a band gap around this frequency opens.

\section{Results}

Our experimental and theoretical results are shown in figure 4 for the cis-polyacetylene chains and in figure 5 for the transpolyacetylene chains. The Green's function calculations (red dashed curves) agree qualitatively with the experimental data (blue solid curves), in particular for short chains. The differences between the resonance frequencies are within the width of the resonances and apart from a few resonances their heights vary on the $50 \%$ level.

\subsection{Transport band gap due to dimerization}

The short chains still show individual resonance peaks, which are located approximately at the eigenenergies of the closed tight-binding Hamiltonian (1). When the chain length is increased, these resonances are less pronounced and two conduction bands arrise, which are separated by a band gap around the frequency $v=0$. The band gap exists in the cis- as well as in the trans-chains but it is more pronounced in the former configuration. This can simply be attributed to the fact that the number of atoms and hence the chain length is much larger in the cis- than in the trans-chains. Numerical calculations confirm that the band gap becomes more pronounced also in the trans-configuration when the chain length is increased.

Additionally, figure 6 shows the experimentally measured transmission through dimerized chains (blue-solid curves) and homogenous chains (red-dashed curves), where the bond lengths are all equal. In this case the chains are composed by units of only one carbon atom (monomer). The homogenous chain does not show a band-gap but a conduction band ranging from $-70 \mathrm{MHz}$ to $90 \mathrm{MHz}$ and hence, confirms that the opening of the band gap is due to the dimerization of the chain. The opening of the band gap due to the dimerization can be understood by considering an infinitely long chain with first nearest
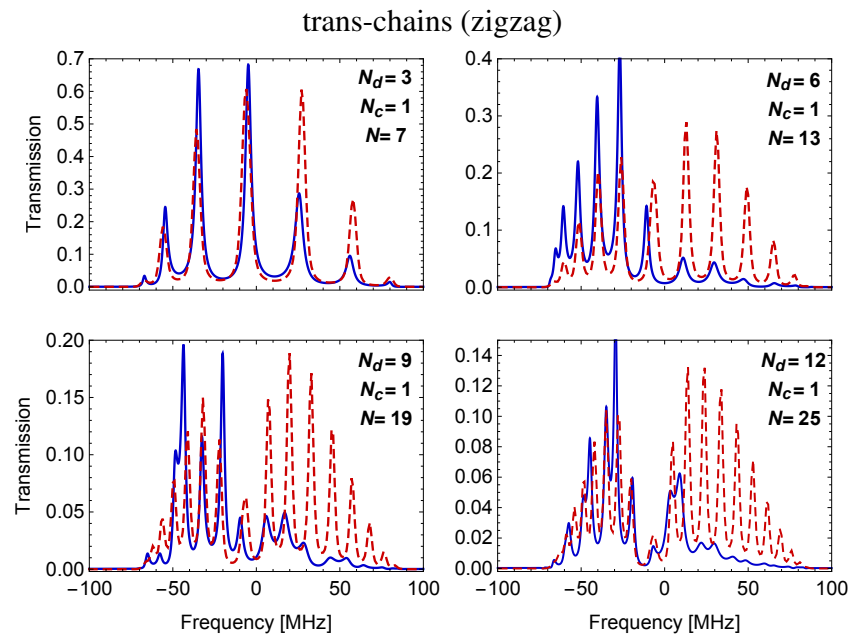

Figure 5: Transmission through trans-polyacetylene chains (zigzag). Experimental data are shown by blue-solid curves, calculations by red-dashed curves. Distinct transmission resonances can be observed. The opening of a band-gap with increasing chain length can be seen only slightly because the total length of the trans-chains is less compared to the cis-chains studied in figure 4
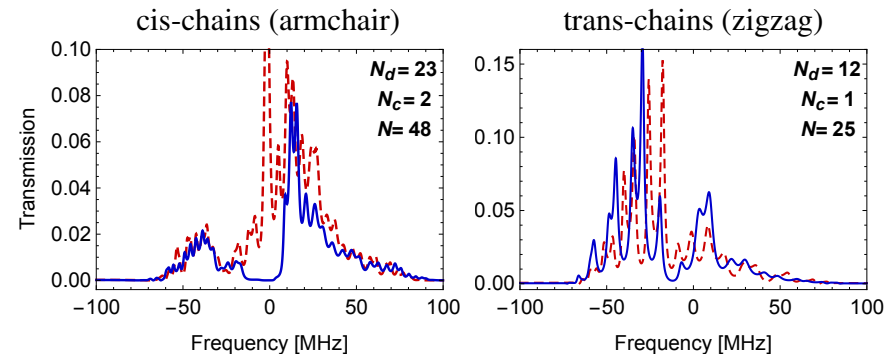

Figure 6: Experimentally measured transmission through dimerized chains (blue solid curves) and homogenous chains (red dashed curves). The experiment shows that the dimerized chains have a band gap, the homogeneous chains not.

neighbor interaction only. Such a first nearest neighbor tightbinding model is known in chemistry also as the Hückel model [36, 37]. The tight-binding Hamiltonian of this chain can be written as

$$
H_{\infty}=\sum_{i=-\infty}^{\infty} t|i, 1\rangle\left\langle i, 2\left|+t^{\prime}\right| i, 2\right\rangle\langle i+1,1|+\text { H.c. }
$$

where $t$ and $t^{\prime}$ are the alternating coupling strengths in the dimerized chain. The quantum states of the dimers are denoted by $|i, 1\rangle$ and $|i, 2\rangle$. Applying a Fourier transform, we obtain a simpler $2 \times 2$ Schrödinger equation for the dimer state in momentum space

$$
\left(\begin{array}{cc}
0 & t+t^{\prime} e^{\mathrm{i} k} \\
t+t^{\prime} e^{-\mathrm{i} k} & 0
\end{array}\right)\left(\begin{array}{l}
k, 1 \\
k, 2
\end{array}\right)=\epsilon(k)\left(\begin{array}{l}
k, 1 \\
k, 2
\end{array}\right) .
$$

Its eigenvalues are

$$
\epsilon(k)= \pm \sqrt{\Delta^{2}+4 t t^{\prime} \cos ^{2}(k / 2)},
$$

where $2 \Delta=2\left|t-t^{\prime}\right|$ gives the band gap. For the chains above, we have $2 \Delta=2|43.9-36.2| \mathrm{MHz} \approx 15.4 \mathrm{MHz}$, which agrees with the gap of around $15 \mathrm{MHz}$ seen in figure 4 and figure 6 for 

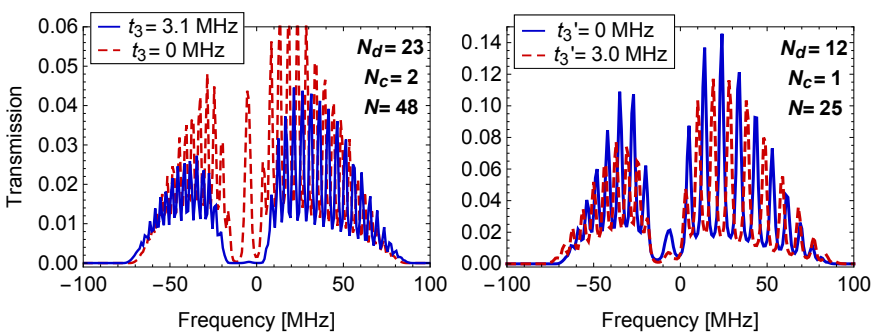

Figure 7: Left: Calculated transmission through the longest studied cispolyacetylene chain. If only couplings up to second nearest neighbors would be taken into account (red-dashed curve), a distinct resonance peak in the center of the band gap would arrise, which is not present if also third nearest neighbors are considered (blue-solid curve) nor in the experimental data (see figure 4). Right: For the trans-chain contrary behavior is observed. If interactions to third nearest neighbors are taken into account by means of figure 3 the small resonance peak at $v=-5 \mathrm{MHz}$ is suppressed which does not agree with the experimental data in figure 5

the experimental data. Have in mind that in the calculation the natural broadening is not taken into account, which leads to an reduction of the observed band gap. Note that the above equations are also valid for the homogenous chain $\left(t=t^{\prime}\right)$, where the band gap $2 \Delta=0$ disappears.

\subsection{Importance of higher-neighbor couplings}

We would like to emphasize that the inclusion of the third neighbor coupling $t_{3}$ is crucial to obtain the gap for the cischain as can be seen in figure 7. If the $t_{3}$ is set to zero in the numerical calculation, a distinct transmission peak occurs at $v=-5 \mathrm{MHz}$, which is neither present in the experiment (see figure 4 (lower right)) nor in the calculation including $t_{3}$ appropriately. On the other hand side for the trans-chain the contrary behavior is observed once we include couplings to third nearest neighbors extracted from figure 3 . The small resonance peak close to $v=-5 \mathrm{MHz}$ is suppressed not agreeing with the experimental observation in figure 5 (lower right). We attribute this to the fact that the coupling is suppressed by the shielding of the other resonators (see table 1).

\subsection{Effect of edge atoms}

Figure 8 shows the calculated transmission through the short chains sketched in the inset. When the chain consists only of dimers without any additional edge atom (i.e. $N_{c}=0$ ) also in short chains a band gap or at least a broad transmission minimum can be observed (black-dotted curves). When an additional edge atom is attached to one of the chain ends $N_{c}=1$, a distinct resonance peak is induced in the center of the band gap around $v=0$ (red-dashed curves). This resonance peak belongs to an exponentially decreasing edge state which is localized at the chain end. Due to its localization this edge state is non-conductive in long chains and the corresponding resonance peak disappears. When edge atoms are attached to both chain ends, two peaks are observed in the transmission (bluesolid curves). These peaks belong to two states which are localized symmetrically or anti-symmetrically at both ends. With increasing chain length the splitting of these peaks and their height decrease, until they finally do not contribute to the transmission any more. The additional resonance peaks due to edge
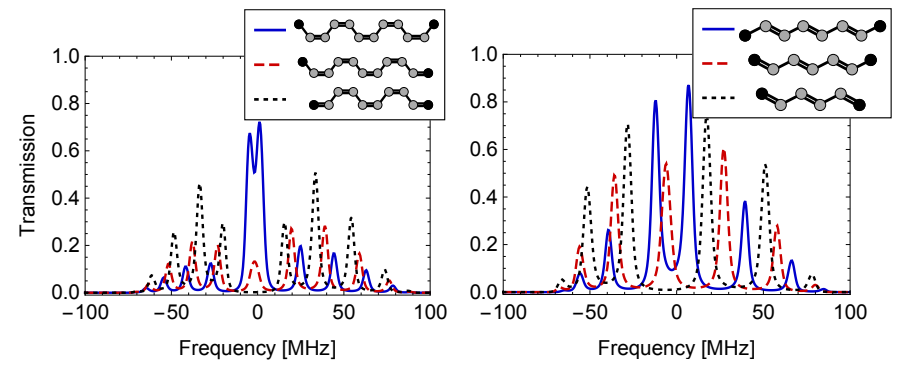

Figure 8: Calculated transmission through the short chains sketched in the inset. In a short chain of dimers (black-dotted curves) a band gap or at least a broad transmission minimum can be observed. When additional edge atoms are attached to the chain ends (red-dashed and blue-solid curves), resonance peaks arrise in the band gap around $v=0$. These peaks are due to two edge state, which are localized symmetrically and anti-symmetrically at both chain ends.

atoms can be seen clearly in the first row of figure 4 and figure 5. Our numerical calculations show that the same effects appear also in pure dimer chains (shown at the bottom of the inset of figure 8) if the contacts (or antennas) are not coupled to the blue shaded atoms but moved one atom inside the chain. Note that the observed resonance peaks are not an even-odd parity effect of the total chain length, which can be observed also in molecular chains [38].

\section{Conclusions}

Summarizing we have presented microwave experiments to emulate the transport in individual polyacetylene chains, where the optimal structure of the molecule has been obtained by the DFT method. The experiments have been accompanied with tight-binding transport calculations using the NEGF method. Both, our experiments and calculations confirm that dimerization produces a band gap in these chains. If the chains are sufficiently long, a band gap appears for cis- and trans-chains for all possible configurations of edge atoms and contact positions. This has been seen experimentally for a single nondimerized edge and has been confirmed numerically for other edge and contact configurations. Thus, returning to the motivation given at the beginning of this article, long polyacetylene chains might indeed replace carbon nanotubes in molecular transistors. Moreover, we have also shown that short dimer chains have a pronounced transmission minimum, if no additional edge atoms are attached and the contacts are at the chain ends. Thus, at the expense of having to know precisely the geometry of the molecule and the way how it is coupled to the contacts, it may well be possible to use rather short chains for novel molecular transistors. While this is probably the first microwave emulation of a molecule that is not largely made up of benzene rings, the actual scope for such microwave emulations is very wide. A first option would be to study local defects, for example, a missing double bond (e.g. due to a substitution of a hydrogen atom) [7, 8, 12]. Such defects produce resonances within the band gap, similar to the observed resonances due to edge atoms. A single defect has been used recently to establish a robust topological mode, which could be used for quantum 
computation [39, 40]. Studies of other conjugated carbon systems using similar experimental methods are in process. The successful emulation of boron-nitrogen sheets [41] suggest that a larger variety of molecules can be studied with similar techniques.

\section{Acknowledgments}

Financial support from CONACyT research grant 219993 and PAPIIT-DGAPA-UNAM research grants IG100616 and IN114014 is acknowledged. T.S. acknowledges a postdoctoral fellowship from DGAPA-UNAM. J.A.F.-V acknowledges financial support from CONACyT project CB2012-180585. T.H.S. and J.A.F.-V. are grateful for the hospitality regularly received at the LPMC. We acknowledge extensive use of the MIZTLI super computing facility of DGTIC-UNAM under project SC15-1-S-20.

\section{References}

\section{References}

[1] J. C. Cuevas, E. Scheer, Molecular Electronics: An Introduction to Theory and Experiment, World Scientific, 2010.

[2] S. J. Tans, A. R. M. Verschueren, C. Dekker, Room-temperature transistor based on a single carbon nanotube, Nature 393 (1998) 49. doi: 10.1038/29954

[3] A. Javey, J. Guo, Q. Wang, M. Lundstrom, H. Dai, Ballistic carbon nanotube field-effect transistors, Nature 424 (2003) 654. doi:10.1038/ nature01797

[4] R. Martel, T. Schmidt, H. R. Shea, T. Hertel, P. Avouris, Single- and multi-wall carbon nanotube field-effect transistors, Appl. Phys. Lett. 73 (1998) 2447. doi:10.1063/1.122477

[5] J.-C. Charlier, X. Blase, S. Roche, Electronic and transport properties of nanotubes, Rev. Mod. Phys. 79 (2007) 677. doi:10.1103/ RevModPhys.79.677

[6] M. M. Shulaker, G. Hills, N. Patil, H. Wei, H.-Y. Chen, H.-S. P. Wong, S. Mitra, Carbon nanotube computer, Nature 501 (2013) 526. doi:10. 1038/nature12502

[7] W. Su, J. Schrieffer, A. Heeger, Solitons in polyacetylene, Phys. Rev. Lett. 42 (1979) 1698. doi:10.1103/PhysRevLett.42.1698

[8] A. J. Heeger, S. Kivelson, J. R. Schrieffer, W. P. Su, Solitons in conducting polymers, Rev. Mod. Phys. 60 (1988) 781. doi:10.1103/RevModPhys . 60.781

[9] A. J. Heeger, Semiconducting and metallic polymers: The fourth generation of polymeric materials, J. Phys. Chem. B 105 (2001) 8475. doi:10.1021/jp011611w

[10] A. Schnurpfeil, M. Albrecht, Charge transport properties of molecular junctions built from dithiol polyenes, Theo. Chem. Acc. 117 (2007) 29. doi:10.1007/s00214-006-0125-1

[11] D. Nozaki, Y. Girard, K. Yoshizawa, Theoretical study of long-range electron transport in molecular junctions, J. Phys. Chem. C 112 (2008) 17408. doi:10.1021/jp806806j

[12] D. Nozaki, H. M. Pastawski, G. Cuniberti, Controlling the conductance of molecular wires by defect engineering, New J. Phys. 12 (2010) 063004. doi:1367-2630/12/6/063004

[13] H. Shirakawa, Synthesis and characterization of highly conducting polyacetylene, Synthetic Metals 69 (1995) 3. doi:10.1016/ 0379-6779(94) 02340-5

[14] H. Shirakawa, Nobel lecture: The discovery of polyacetylene film ${ }^{-}$the dawning of an era of conducting polymers, Rev. Mod. Phys. 73 (2001) 713. doi:10.1103/RevModPhys.73.713

[15] M. Bellec, U. Kuhl, G. Montambaux, F. Mortessagne, Topological transition of dirac points in a microwave experiment, Phys. Rev. Lett. 110 (2013) 033902. doi:10.1103/PhysRevLett.110.033902
[16] M. Bellec, U. Kuhl, G. Montambaux, F. Mortessagne, Tight-binding couplings in microwave artificial graphene, Phys. Rev. B 88 (2013) 115437. doi:10.1103/PhysRevB.88.115437

[17] P. Hohenberg, W. Kohn, Inhomogeneous electron gas, Phys. Rev. 136 (1964) B864-B871. doi:10.1103/PhysRev.136.B864

[18] W. Kohn, L. J. Sham, Self-consistent equations including exchange and correlation effects, Phys. Rev. 140 (1965) A1133-A1138. doi:10. 1103/PhysRev.140.A1133

[19] R. Ditchfield, W. J. Hehre, J. A. Pople, Self-consistent molecular-orbital methods. ix. an extended gaussian-type basis for molecular-orbital studies of organic molecules, J. Chem. Phys. 54 (1971) 724. doi:10.1063/1. 1674902

[20] A. D. Becke, Density-functional exchange-energy approximation with correct asymptotic behavior, Phys. Rev. A 38 (1988) 3098. doi:10. 1103/PhysRevA.38.3098

[21] M. J. Frisch et al., GAUSSIAN 09 (Revision A.02), Gaussian, Inc., 2009.

[22] R. G. Parr, W. Yang, Density Functional Theory of Atoms and Molecules, Oxford University Press, 1989.

[23] D. S. Sholl, J. A. Steckel, Density Functional Theory, Wiley, 2009.

[24] H. Teramae, Ab initio studies on the cis-trans energetics of polyacetylene, J. Chem. Phys. 85 (1986) 990. doi:10.1063/1.451256

[25] L. Rodríguez-Monge, S. Larsson, Conductivity in polyacetylene. i. ab initio calculation of charge localization, bond distances, and reorganization energy in model molecules, J. Chem. Phys. 102 (1995) 7106. doi:10.1063/1.469104

[26] R. E. Peierls, Quantum Theory of Solids, Clarendon Press, Oxford, 1955.

[27] J. A. Franco-Villafañe, E. Sadurní, S. Barkhofen, U. Kuhl, F. Mortessagne, T. H. Seligman, First experimental realization of the dirac oscillator, Phys. Rev. Lett. 111 (2013) 170405. doi:10.1103/PhysRevLett. 111.170405

[28] E. Sadurní, J. A. Franco-Villafañe, U. Kuhl, F. Mortessagne, T. H. Seligman, Schematic baryon models, their tight binding description and their microwave realization, New J. Phys. 15 (2013) 123014. doi:10.1088/ 1367-2630/15/12/123014

[29] W. Barford, Electronic and optical properties of conjugated polymers, Oxford University Press, 2005.

[30] M. Geoghegan, G. Hadziioannou, Polymer Electronics, Oxford Univerity Press, 2013.

[31] S. Kivelson, Electron hopping conduction in the soliton model of polyacetylene, Phys. Rev. Lett. 46 (1981) 1344. doi:10.1103/ PhysRevLett.46.1344

[32] D. Baeriswyl, K. Maki, Electron correlations in polyacetylene, Phys. Rev. B 31 (1985) 6633-6642. doi:10.1103/PhysRevB.31.6633

[33] A. Troellsch, F. Temps, Analysis of vibrationally highly excited bound and resonance states of dco $\left(x^{2} a^{\prime}\right)$ using an effective polyad hamiltonian, Z. Phys. Chem. 215 (2001) 207. doi:10.1524/zpch.2001.215.2. 207

[34] C. Jung, H. S. Taylor, E. Atılgan, Extraction of the vibrational dynamics from spectra of highly excited polyatomics: Dco, J. Phys. Chem. A 106 (2002) 3092-3101. doi:10.1021/jp014008m

[35] S. Datta, Electronic Transport in Mesoscopic Systems, Cambridge University Press, 1997.

[36] E. Hückel, Quantentheoretische Beiträge zum Benzolproblem, Zeitschrift für Physik 70 (1931) 204. doi : 10.1007/BF01339530

[37] J. Murrell, S. Kettle, J. Tedder, The Chemical Bond, Wiley, 1985.

[38] T.-S. Kim, S. Hershfield, Even-odd parity effects in conductance and shot noise of metal-atomic-wire-metal (superconducting) junctions, Phys. Rev. B 65 (2002) 214526. doi : 10.1103/PhysRevB .65.214526

[39] H. Schomerus, Topologically protected midgap states in complex photonic lattices, Optics Letters 38 (2013) 1912. doi:10.1364/OL.38. 001912

[40] C. Poli, M. Bellec, U. Kuhl, F. Mortessagne, H. Schomerus, Selective enhancement of topologically induced interface states in a dielectric resonator chain, Nat. Com. 6 (2015) 6710. doi:10.1038/ncomms7710

[41] S. Barkhofen, M. Bellec, U. Kuhl, F. Mortessagne, Disordered graphene and boron nitride in a microwave tight-binding analog, Phys. Rev. B 87 (2013) 035101. doi:10.1103/PhysRevB.87.035101 